\title{
SALUD-FAMILIA-ENVEJECIMIENTO POBLACIONAL, UNA TEMÁTICA QUE LLAMA LA ATENCIÓN DE LAS CIENCIAS SOCIALES
}

\author{
HEALTH-FAMILY- POPULATION AGING, A THEME THAT CALLS THE ATTENTION \\ OF SOCIAL SCIENCES
}

\begin{abstract}
RESUMEN
Las autoras se acercan a una arista de un problema que en las últimas décadas reclama la atención y mirada propositiva de varios sectores de la sociedad, en Cuba y el resto del mundo. Se exponen los principales paradigmas, teóricos y escuelas de pensamiento que han estudiado la familia, la salud, y la relación entre ellas, para ello se sistematizan, resultados de investigaciones anteriores sobre las materias, se analizan limitaciones y aportes de cada propuesta. Se requirió el empleo de métodos de obtención de información del nivel teórico, el objetivo trazado es el de sistematizar los aspectos teóricos que han tributado, desde las ciencias sociales, a la comprensión del papel de la familia y el enfoque sociológico de salud en los estudios comunitarios. Todo ello a través de la consulta a textos relacionados con el tema y que gozan de actualidad. El interés de las autoras más allá del objetivo reconocido es llamar la atención sobre la responsabilidad social de la familia y de la comunidad en general, en los momentos en que Cuba envejece y necesita una mirada proactiva para enfrentar tal reto, aspecto que es parte de las conclusiones del estudio: el tema compete a toda la sociedad.
\end{abstract}

Palabras clave: envejecimiento; familia; salud.

\section{ABSTRACT}

This paper only intends an approach to an edge of the problem, which in recent decades demands attention, and a diligent and purposeful look of various sectors of the society, especially in Cuba. The work presents the main theoretical paradigms and schools of thought that have studied the family, the definitions of health, and the relation among them. The results of research done on these issues are also systematized, and constraints and theoretical contributions of each proposal are analyzed. An analysis of the main theoretical traditions on family-related health is carried out, and for this aim, a definition of family is given. Methods of obtaining information at theoretical level were used, and the settled goal is to systemize the theoretical aspects that have been offered from social sciences to understand the role of the family and the sociological approach to health in the community studies. All this through consultation of current texts related to the subject. The interest of the authors, beyond the declared aim, is to draw attention to the social responsibility of the family and the community at large with the issue of health, in a time when Cuba is aging and needs a proactive look to face this challenge.

Keywords: aging; family; health.
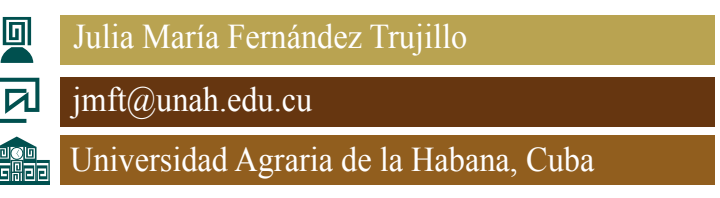

\section{Ailen Bernardo Romay}

ailen.bernardo@dmayabeque.icrt.cu

Delegación Provincial de Radio y Televisión Mayabeque, Cuba 


\section{INTRODUCCIÓN}

El fenómeno del envejecimiento poblacional alcanza cada vez mayores dimensiones, en el mundo y en particular en Cuba, a consecuencia de una marcada disminución en la fecundidad y un incremento significativo de la esperanza de vida al nacer (Benítez y Alfonso 2010). Según cálculos realizados a partir de datos nacionales, en el año 2011 la población envejecida en el país representaba ya un $18.1 \%$ del total poblacional (Anuario Demográfico 2011). Aspecto significativo para ser tomado en cuenta.

Ello trae consigo la necesidad de implementar medidas sociales para enfrentar dicho fenómeno. Uno de los sistemas que advierte significativos retos en este sentido es el sector de la salud el que debe velar y garantizar la calidad de vida de las personas envejecidas. Entre las dificultades que influyen en el buen desarrollo de la atención del adulto mayor se encuentran: la falta de recursos humanos y materiales, la deficiente autogestión y el hecho de no tomar en consideración las necesidades reales y sentidas de este grupo poblacional expresadas desde sus propias voces (Briceño-León 2003).

De estas ideas se desprende que la concientización acerca de la responsabilidad familiar ante la salud permite reactivar el sentimiento de pertenencia hacia la comunidad y genera cultura de acción colectiva, así como afrontamientos familiares favorecedores a la salud (Louro 2011). A partir del criterio de este autor resulta evidente, la importancia que poseen las familias y las comunidades como los entornos en los cuales se establece por primera vez el comportamiento saludable y donde se moldean inicialmente la cultura, los valores y las normas sociales. Pero el tema no solo puede y debe ser abordado desde un ángulo, para su cabal comprensión es necesario una mirada más amplia.
Es por tal razón que este artículo tiene como objetivo: sistematizar los aspectos teóricos que han tributado, desde las ciencias sociales, a la comprensión del papel de la familia y el enfoque sociológico de salud en los estudios comunitarios, de manera tal que permita mostrar la significación que tiene para el momento actual, la atención a este sector poblacional desde una postura multifactorial e interdisciplinar.

\section{METODOLOGÍA}

Durante el estudio, las autoras mostraron claro interés en buscar desde la ciencia y estudios precedentes los vínculos entre familia y salud para la atención a un sector poblacional que cada vez se va haciendo mayor y reconociendo que la atención a ese grupo etario tiene un enfoque multisectorial.

Los métodos de obtención de información empleados, fundamentalmente, fueron del nivel teórico, entre los que están el histórico lógico, que facilitó buscar las relaciones e historicidad del estudio; el análisis -síntesis para llegar a concreciones respecto a las relaciones categoriales; el sistémico estructural que facilitó establecer esas relaciones entre la salud y la familia. Esos métodos empleados en el estudio permitieron llegar a conclusiones, tales como que, el hecho ha sido abordado desde la sociología, la salud, la psicología, entre otras ramas y que el contexto actual demanda de una visión más integral del concepto de salud pública, así como la necesidad latente para que la población en general se reconozca como agente promotor de salud y en específico la familia juega un rol trascendental en la atención a los gerontes. Con ello fueron aclaradas las expectativas de las autoras. 


\section{RESULTADOS DEL ESTUDIO, DEFI- NICIONES Y ANÁLISIS}

La salud tiene una particularidad y es que desde sus orígenes no pudo definirse por sí misma, sino que fue entendida por oposición a la enfermedad. Tal fue el caso de Galeno, quien señalaba que salud "es lo que se posee cuando uno puede moverse sin dolor;" (Domínguez 2001:10) mientras que mucho tiempo después, en el siglo XVIII, Samuel Johnson indicaba que salud consistía en estar carente de malestar, dolor o enfermedad. Con estas dos concepciones de salud, bastante distantes en el tiempo una de otra, se puede llegar a la definición que ha predominado en la historia de la humanidad y ésta es: "ausencia de enfermedad" (Domínguez 2001:10).

No es hasta el siglo XIX, que se logra comprender que la salud es un fenómeno multifactorial y que debe ser visto desde su multidimensionalidad. Entre los teóricos que incorporan estas nuevas ideas se encuentra Engels con su conocido estudio sobre las condiciones de la clase obrera en Inglaterra. En esta obra establece que Alemania se constituiría como el primer país con un seguro médico y sentaría el precedente para dar cobertura al derecho a la salud. De igual manera, pone de manifiesto la relevancia de los factores sociales para la salud humana (Beldarraín 2006).

Llegado, en el siglo XX, se comienza hablar del vínculo estrecho entre las ciencias de la salud y las ciencias sociales, debido a la aparición de las escuelas con un enfoque cultural e histórico, donde la subjetividad emerge y comienza a transitarse hacia la validación de paradigmas más bien culturalistas. La concepción acerca de la salud comienza a variar y los aportes de las ciencias sociales: de la sociología, la antropología y la psicología se hacen cada vez más influyentes en la comprensión del ser humano. El hombre empieza a concebirse como un ser integral y la enfermedad y la salud responden, por tanto, a un proceso en el que influyen variados ámbitos de la vida del ser humano.

Las concepciones médicas de salud se basan en el punto de vista de la medicina como actividad profesional y se dividen, a su vez, en tres apartados de acuerdo a la práctica médica: la salud somática-fisiológica, la psíquica y la sanitaria.

En la concepción somática-fisiológica la salud es el bienestar del cuerpo y del organismo físico y la enfermedad es el proceso que altera este bienestar (Muñoz 2007). Dicha concepción ha permanecido durante la mayor parte de la historia más pendiente de la enfermedad que de la salud, pues no fue hasta el pasado siglo que comenzó a estudiarse como un estado positivo al que hay que acceder a través de prácticas preventivas, como algo más complejo e importante que la mera ausencia de enfermedad o la enfermedad compensada.

La concepción psíquica por su parte, se opone tradicionalmente a la orgánica como lo subjetivo a lo objetivo, pero sin obviar la estrecha interrelación existente en el organismo humano entre cuerpo y psique (Moragas 1976). Resulta importante destacar que, a pesar de los aportes realizados por Freud y las más modernas interpretaciones de la personalidad, la limitación fundamental de esta concepción psíquica de salud radica en la subjetividad e indeterminación de sus manifestaciones, en comparación con la dolencia somática, ya que el enfermo puede siempre afirmar que no tiene salud, aunque objetivamente el médico no tenga base para aceptarlo.

La concepción sanitaria se centra en el mantenimiento, la preservación y la recupera- 
ción de la salud, enfatizando en la salud colectiva de una población o comunidad, es decir una consideración colectiva de la salud. En este sentido, dicha concepción estudia problemas sociales y sanitarios como el control de la población, la ancianidad, la delincuencia juvenil y la deficiencia mental. Se interesa por los grupos sociales y posee un gran enfoque preventivo.

Desde otro ángulo la salud pública se torna en la ciencia y el arte de prevenir las dolencias y las discapacidades, prolongar la vida, fomentar la salud y la eficiencia física $y$ mental. Todo ello se realiza con los esfuerzos organizados de la comunidad para educar al individuo en los principios de la higiene personal, organizar los servicios para el diagnóstico, el tratamiento y la rehabilitación. Además desarrolla la maquinaria social que le asegura a cada miembro de la comunidad un nivel de vida adecuado para el mantenimiento de la salud (Organización Mundial de la Salud 1996).

Más avanzado el tiempo, la Organización Mundial de la Salud (OMS), plantea que la salud es "un estado de bienestar físico, mental y social completo y no meramente la ausencia de enfermedad o disminución" (Organización Mundial de la Salud 2015). Aspecto que resulta interesante para el objetivo que se persigue en este artículo, puesto que se reconoce el amplio espectro que debe identificar a la salud, la que no puede entenderse como un estado fijo a alcanzar mediante la supresión de los agentes patógenos, pues se estaría negando la propia evolución física, psíquica y social. La concepción de la OMS posibilita a la práctica médica y sanitaria trazarse nuevos objetivos para mejorar los estados parciales de salud física, psíquica o social.

Resulta válido señalar entonces, luego de este recorrido, sobre las distintas definicio- nes de salud que no se puede sustraer del carácter eminentemente social que la compone.

Es en este entramado en que el Estado se erige en el principal actor institucional de la salud pública y en una entidad individualizada desde el punto de vista operativo que tiene personalidad jurídica e instrumentos de actuación propios y poderosos que les permite actuar en consecuencia al contar con instituciones responsables directas del sector de la salud como el propio Ministerio de Salud. Una de las funciones más importantes del Estado, en este sentido, es la movilización de la sociedad civil y la capacitación de la población para la participación social.

Las posibilidades y estrategias para enfrentar la atención a estas personas con la calidad y excelencia que merecen se circunscriben a los Consultorios Médicos de la Familia, los hogares de ancianos, los círculos de abuelos, las casas de abuelos y las salas de geriatría de los hospitales. Aunque no hay dudas que los programas que se utilizan en la actualidad cuentan con un alto prestigio por su calidad, es válido señalar que no tratan con la debida suficiencia lo relacionado al cuidado y la atención de los adultos mayores, ni la dinámica familiar y social que dicho proceso presenta. En su mayoría son programas que aborda una arista del problema, la fisiológica.

La intersectorialidad, por su parte, constituye un enfoque que sintetiza los más recientes presupuestos de la ciencia al contemplar los preceptos del pensamiento sistémico como fundamento teórico para actuar con más acierto y eficacia en circunstancias complejas. Aboga por la acción coordinada de diversos sectores de la sociedad o parte de ellos en función de solucionar un problema de salud o actuar sobre sus factores determinantes, condicionantes y desencadenantes 
(Castell 2010). Este enfoque reconoce a cada comunidad como escenario de procesos singulares de producción de salud que requieren intervenciones ajustadas a sus características. Aboga además por la necesidad de establecer relaciones horizontales y participativas, con alto grado de compromiso entre los diversos sectores.

Resulta entonces, que son varias las instituciones las que deben "ponerse de acuerdo" en el establecimiento de esa estrategia de atención a la salud. Al analizar el soporte en que se levanta la atención a la salud se percibe a la familia como institución y grupo social que puede contribuir a la atención familiar desde esa visión multisectorializada e interdisciplinar.

La familia no es más que una estructura jerarquizada de papeles, un subsistema social que funciona como institución $\mathrm{y}$ grupo social, cuyas relaciones entre sus miembros se basan en el parentesco, en la residencia común y su funcionamiento como unidad doméstica (Fleitas 2005), como se puede observar la interpretación de familia que se asume no es solo la mirada primigenia emanada de la escuela positivista, aquí se aboga por una visión más amplia que habla de la interacción.

Los principales estudios que han abordado la relación familia-salud responden esencialmente a investigaciones realizadas en el campo de la medicina. En este sentido, se estudiaron los procesos de salud más allá de los problemas físicos, biológicos y ambientales, reconociendo el papel de los fenómenos de origen social y psicológico y el papel de determinados grupos sociales como la familia (Gaminde 1997). Algunos representantes del movimiento de la Medicina Social, tales como Breilh y Castellanos, resaltaron la influencia de los determinantes sociales en la salud de la población y para ello se basaron en la filosofía materialista dialéctica y en la concepción integral biosocial del hombre (Morales 1997).

Otra de las ciencias que ha estudiado esta temática ha sido la psicología. En esta rama destacan figuras como Pérez Lovelle, psicólogo cubano que analizó la determinación de la salud según varios niveles, colocando a la familia en el nivel intermedio (Castell 2007) y de hecho reconoce que entran a ocupar un papel importante en el desenvolvimiento de las personas y su desarrollo las interacciones y las relaciones familiares y grupales.

La Escuela histórico - cultural desarrollada por L. S. Vygotsky sobre la base del materialismo dialéctico e histórico, ofreció también una concepción teórica que reconoce la influencia de las mediaciones sociales mediante los contextos y grupos con los cuales se relaciona el individuo en toda su vida (Rojo y García 2000). Para el marxismo, la génesis del hombre tiene su fuente y origen en la interacción entre el individuo, su grupo social y su medio natural y se destaca el papel de la familia como potenciadora del desarrollo.

En la familia se transmite la herencia cultural y es el lugar donde cada generación deja plasmada su historia, su experiencia, sus valores, sus costumbres y principios; aspectos relacionados con el proceso educativo básico de la producción de la salud. En este sentido, autores como Domínguez plantean que lo social es atravesado por diferentes ejes que constituyen momentos de mediatización: el eje de la sociedad y la cultura y el eje de lo grupal y lo histórico individual (Domínguez 2001). Se refiere a la familia como uno de esos agentes sociales mediadores del aprendizaje individual y grupal. Según este autor, debido a la alta presencia que la familia tiene en las primeras etapas de formación de la personalidad, se constituye 
en uno de los mediadores fundamentales de todas las influencias valorativas.

En lo referente a la conservación y protección de la salud, la familia cumple roles esenciales y de gran trascendencia, en tanto desempeña las funciones inherentes a la satisfacción de necesidades básicas, el cuidado de sus integrantes, lo que incluye además la obtención y administración de los recursos económicos para el hogar y la organización de servicios domésticos. Es en la familia donde se forman los motivos, patrones y hábitos relacionados con el comportamiento implicado en la salud, se gestan procesos que actúan como sus protectores o desencadenantes de la enfermedad y se desarrollan recursos de apoyo altamente significativos y efectivos.

Las relaciones familiares intervienen en la generación y evolución de síntomas de enfermedad. Esta idea es defendida por investigadores como Dingle que se dedicaron al análisis de familias para estudiar la incidencia de algunas enfermedades y obtuvieron que éstas variaban en dependencia de la intensidad y magnitud de las relaciones familiares (Donati 1994), según el autor la fragmentación y la desorganización de la familia son factores altamente condicionantes en la salud.

Varios resultados de investigaciones han documentado la relación del funcionamiento familiar como factor predisponente o coadyuvante en la producción y curso de diversos problemas de salud en sus integrantes según expresa Risteen (2006).

Estudios realizados en Cuba, específicamente, han evidenciado que las familias disfuncionales tienen gran incidencia en enfermos crónicos y en problemas de alcoholismo. También expresan que condicionan conductas antisociales, promiscuidad y suicidios (Rojo y García 2000).
La aparición de un problema de salud en uno de los miembros de la familia propicia la inclusión de un plan hospitalario en el sistema familiar. La familia colabora en el período de la rehabilitación y en la adaptación a las secuelas que dejó la enfermedad. La ayuda instrumental y emocional que necesita el afectado, la adecuación del hogar, de la economía, la redistribución de roles y la colaboración en la reinserción a la sociedad, las cubre la familia.

En la Facultad de Psicología de la Universidad de La Habana desde hace más de 15 años se estudia a la familia desde una perspectiva psicosocial, describiendo su influencia en las transformaciones sociales. Arés considera imprescindible el análisis de variables socio-psicológicas que expresen la incidencia de lo social (cultura), ideas, principios, normas y valores sociales en lo individual y las repercusiones psicológicas (Arés 1990).

Uno de los aspectos a tener en cuenta en la relación familia-salud es la conexión existente entre las condiciones materiales de vida que posee la familia y su estado de salud. En nuestro país existen diversos centros de estudios que se han dedicado a realizar investigaciones sobre esta temática, tales como la Facultad Latinoamericana de Ciencias Sociales de Cuba de la Universidad de La Habana, el Instituto de Antropología y el Grupo de Familia del Centro de Investigaciones Psicológicas y Sociológicas (Artiles 2007).

También han desarrollado estudios en este sentido diferentes investigadores cubanos como María Elena Astraín y María del Carmen Pría, bioestadísticas de formación y profesoras de la Facultad de Salud Pública, quienes destacan cómo las condiciones materiales precarias de una familia influyen en las estrategias de salud que adoptan 
(Astraín 2004). Sin embargo, los análisis contenidos en dichos estudios van más allá de la dicotomía familia-salud, pues tienen en cuenta también indicadores como pobreza, desarrollo y desigualdades de género.

Para los fines de la atención de salud, se requiere una investigación de carácter holístico que recoja las formas en las que se materializa en la familia la influencia de las condiciones económicas y sociales, así como las características concretas de expresión de los procesos psicológicos resultantes de la interacción entre sus miembros y de sus formas de desarrollo. Debe permitir la caracterización del funcionamiento social de la familia, el pleno desarrollo de sus miembros y las necesidades de atención de salud.

La familia interviene en las decisiones sobre el uso de servicios profesionales y constituye la red de apoyo más potente y eficaz en el ajuste a la vida social y ante los procesos de salud (Louro 2011). No obstante, no ha estado bien posicionada en el campo de la salud y su carácter de agente mediador no se ha tenido en cuenta suficientemente en las estrategias de promoción de salud.

Según los criterios de la OMS, uno de los fines principales de cada país debe ser incrementar el nivel de salud de la población, por lo que propone a la familia como uno de los grupos fundamentales en este terreno.

Para ello es preciso entender la salud familiar como la dinámica interna relacional en el cumplimiento de las funciones para el desarrollo de sus integrantes y en la capacidad de enfrentar los cambios del medio social y del propio grupo, propiciando el desarrollo y crecimiento individual según las exigencias de cada etapa de la vida (Pérez y Carmona 1982).
En la década de 1970, siguiendo los principios de la Declaración de Alma-Ata, el Sistema de Salud cubano elaboró un nuevo modelo de atención primaria a la salud. Con este modelo surge el Policlínico Comunitario, que no negaba el anterior modelo, sino que mantenía sus principios e incorporaba nuevos procedimientos (Delgado 1996). Ya todos los países del mundo habían definido de manera unánime en la Declaración de Alma-Ata que la atención primaria de salud es la estrategia clave para alcanzar la meta social de salud para todos (Castell 2010).

Así es como a principios de la década de 1980, surgen las primeras ideas de crear un nuevo modelo de atención comunitaria, con profesionales que pudieran integrar, controlar y coordinar el cuidado de la salud de las personas y las familias (Castell 2010). Se pone a la familia en el eje de las acciones y se esboza el modelo del Médico y la Enfermera de la Familia. Se convierte al médico general en un médico especialista, con formación sólida y con un papel especial en la comunidad.

Desde entonces se asumió la atención primaria como la forma más adecuada para enfrentar los problemas de salud, entendidos éstos como problemas sociales e inseparables del desarrollo socioeconómico de un país.

Sin embargo, se hace necesario señalar que para el logro de esta estrategia se requieren procesos fundamentales en todos los niveles del sistema de salud, en sus instituciones, en sus servicios y, sobre todo, en la mentalidad y actitudes de toda la población, incluyendo a los proveedores de los servicios de salud. 


\section{CONCLUSIONES}

La atención primaria de salud no debe considerarse como una forma de satisfacción parcial de las necesidades sociales de una comunidad pobre, su esencia está en los procesos que surgen de los principios que se reconocen y pueden sintetizarse en la participación, la descentralización, la acción multisectorial, el uso y empleo de las tecnologías apropiadas, es en este marco referencial en que al analizar el fenómeno desde una postura sociológica no debe restringirse la responsabilidad a las instituciones de salud o al Estado y su influencia (educativa/coercitiva) como únicos responsables de este fenómeno si no que tal como lo reconocen estudiosos mencionados con anterioridad la comunidad debe tener este fenómeno en su centro de acción, al igual que se demanda de una visión más integral del concepto de salud pública, expuesto con anterioridad donde toda la población se reconozca como agente promotor de salud y como parte fundamental en ello: la familia.

Finalmente, es necesario, porque cada vez tendemos a ser una población más envejecida, que se produzcan no solo estudios si no acciones concretas de carácter integral que involucren a personal que labora en áreas de salud, la familia, a los y las gerontes y a la comunidad toda en función de la atención de este grupo y vaya creándose una cultura favorecedora de una vida más completa y sana.

\section{REFERENCIAS}

\section{BIBLIOGRÁFICAS}

Anuario Demográfico de Cuba (2011). Oficina Nacional de Estadísticas e Información de Cuba. Cuba: Panorama demográfico.

Arés, P. (1990). Mi familia es así. Investigación psicosocial. La Habana, Cuba: Ed. Ciencias Sociales.

Artiles, L. (2007). Las condiciones de vida como determinantes del proceso salud-enfermedad en la familia: Un enfoque desde lo social. La Habana, Cuba: Editorial Científico Técnica.

Astraín, M. (2004). Situación de salud según condiciones de vida: análisis de Situación de Salud. Anuario de Psicología, (81), 89-104.

Beldarraín, E. (2006). Los médicos y los inicios de la antropología en Cuba. La Habana, Cuba: Fundación Fernando Ortiz.

Benítez, M. \& Alfonso de Armas, M. (2010). La familia como categoría demográfica. La familia y las ciencias sociales, (17), 162-194.

Briceño-León, R. (2003). Las ciencias sociales y la salud: un diverso y mutante campo teórico. Ciencia y salud colecti$v a,(8), 33-45$.

Castell, P. (2010). Intersectorialidad en salud: fundamentos y aplicaciones. La Habana, Cuba: Editorial Ciencias Médicas.

Castell, P. (2007). La intersectorialidad en la práctica social. La Habana, Cuba: Editorial Ciencias Médicas.

Domínguez, M. (2001). Concepto de salud y enfermedad. Revista Cubana Salud Pública, 26 (2), 91-100.

Donati, P. (1994). Sociología de la Salud. Madrid, España: Díaz de Santos.

Fleitas, R. (2005). Sociología y políticas de salud. Selección de Lecturas. La Habana, Cuba: Editorial Félix Varela. 


\section{Unach}

Gaminde, I. (1997). Sociología y Salud. Madrid, España: Editorial S.A. IMC\&C.

Louro, I. (2011). Enfoque familiar en el análisis de la situación de salud. Revista Cubana de Higiene y Epidemiología, 49(2), 151-153.

Moragas, R. (1976). Concepciones de la salud. Revista de Sociología y Medicina, (5), 31-54.

Morales, F. (1997). La Psicología y los Servicios de Salud. Experiencia de trabajo en Cuba. Facultad de Psicología, 13(2), 51-84.

Muñoz, S. (2007). El concepto de Salud: una mirada desde sus orígenes. Revista Cubana Salud Pública, 33(1).

Organización Mundial de la Salud. (2015). Datos sobre el envejecimiento y la salud. Recuperado de http://www.who.int/features/factfiles/ageing/es/

Organización Mundial de la Salud. (1996). Concepto de Salud. Recuperado de http://concepto.de/salud-segun-la-oms/

Pérez, N. \& Carmona, A. (1982). La familia y el nivel de salud de una comunidad. Aspectos conceptuales y metodológicos. Revista Cubana de Administración en Salud, 8(3), 224-241.

Risteen, B. (2006). Envejecimiento y salud. La Habana, Cuba: Editorial Ciencias Médicas.

Rojo, N. \& García, R. (2000). Sociología y salud. Reflexiones para la acción. Revista Cubana de Salud Pública, 24(2), 91-100.

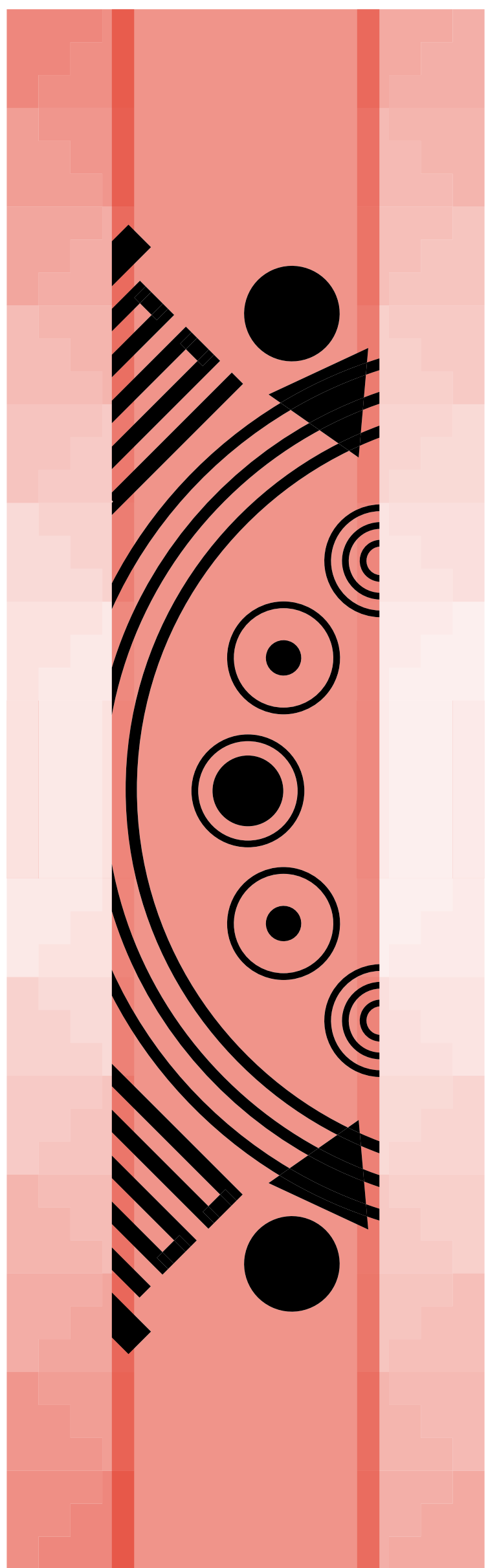

